Artigo Original

Original Article

Lucia Kazuko Nishino ${ }^{1}$ (D) Guilherme Dias Rocha ${ }^{2}$ (1) Thiago Silva Almeida de Souza ${ }^{3}$ (1) Fernando de Andrade Quintanilha Ribeiro ${ }^{4}$ (c)

Pedro Luis Cóser ${ }^{5}$

Descritores

Equilíbrio Postural

Tontura

Vertigem

Valores de Referência

Propriocepção

Keywords

Postural Balance

Dizziness

Vertigo

Reference Values

Proprioception

Endereço para correspondência:

Lucia Kazuko Nishino

R. Jaguaribe, 355, Vila Buarque, São

Paulo (SP), Brasil, CEP: 01224-001.

E-mail: lucianishino@gmail.com

Recebido em: Dezembro 06, 2019

Aceito em: Junho 15, 2020

\section{Protocolo para posturografia estática com provas dinâmicas em indivíduos sem queixas vestibulares utilizando o sistema Horus}

\author{
Protocol for static posturography with \\ dynamic tests in individuals without vestibular \\ complaints using the Horus system
}

\begin{abstract}
Purpose: To propose a protocol for investigating the body balance and determining reference values in different age groups and gender, using the methodology of static posturography with dynamic tests, in low-cost Brazilian equipment for diagnosing balance. Methods: The objectives of this study aimed to propose a protocol for investigating the body balance and determining reference values in different age groups and gender, using the methodology of static posturography with dynamic tests, in new low-cost Brazilian equipment for diagnosing balance. Method: 297 healthy volunteers, between 20 and 89 years old, without vestibular complaints, were divided into six groups according to age group and gender. Stability limits and seven sensory conditions named from $\mathrm{C} 1$ to $\mathrm{C} 7$ were evaluated. The work was carried out using Horus equipment, manufactured in Brazil. Results: The reference values of the stability limit for females and males were obtained according to the ages: from 20 to $59\left(\geq 12,594 \mathrm{~mm}^{2}\right.$ and $\left.\geq 19,221 \mathrm{~mm}^{2}\right)$; from 60 to $69\left(\geq 7,031 \mathrm{~mm}^{2}\right.$ and $\left.\geq 12,161 \mathrm{~mm}^{2}\right)$; from 70 to $89\left(\geq 6,340 \mathrm{~mm}^{2}\right.$ and $\left.\geq 8,794 \mathrm{~mm}^{2}\right)$. For sensory integration tests under conditions $\mathrm{C} 1$ to $\mathrm{C} 7$, as age increased, the values of the Confidence Ellipse (CE) area also increased. Reference percentile values were established for Residual Functional Balance (RFB) and Sensory Analysis (SA). Conclusion: A protocol was established to investigate body balance via static posturography as well as reference values for normal individuals were determined, according to the different gender and age groups.
\end{abstract}

Trabalho realizado no setor de Fonoaudiologia, Irmandade da Santa Casa de Misericórdia de São Paulo - São Paulo (SP), Brasil.

${ }^{1}$ Irmandade da Santa Casa de Misericórdia de São Paulo, Faculdade de Ciências Médicas da Santa Casa de São Paulo - São Paulo (SP), Brasil.

${ }^{2}$ Centro de Audiologia e Pesquisa em Equilíbrio - CAPE - Rio de Janeiro (RJ), Brasil.

${ }^{3}$ Clínica ICL Otorrino - Goiânia (GO), Brasil.

${ }^{4}$ Faculdade de Ciências Médicas da Santa Casa de São Paulo - São Paulo (SP), Brasil.

${ }^{5}$ Clínica Cóser de Otorrino - Santa Maria (RS), Brasil.

Fontes de financiamento: nada a declarar.

Conflito de interesses: nada a declarar

cc) (i) Este é um artigo publicado em acesso aberto (Open Access) sob a licença Creative Commons Attribution, que permite uso, distribuição e reprodução em qualquer meio, sem restrições desde que o trabalho original seja corretamente citado. 


\section{INTRODUÇÃO}

Para um equilíbrio corporal adequado, é necessária a complexa integração entre os sistemas sensorial e motor, permitindo a manutenção de uma postura estável, definindo o equilíbrio estático ou em movimento e constituindo o equilíbrio dinâmico de forma harmônica e precisa. O comprometimento postural do indivíduo pode decorrer de alteração proprioceptiva, ou seja, da percepção da postura e da movimentação do corpo; de alteração vestibular, desencadeada pela posição e/ou o movimento da cabeça; ou de alteração visual, por relações espaciais ${ }^{(1-9)}$.

A posturografia, também denominada estabilometria ou estabilografia, é um teste no qual se usa uma plataforma de força para avaliação geral do equilíbrio, obtendo uma aproximação quantitativa das oscilações do centro de gravidade (CG) do indivíduo nela posicionado. O centro de pressão $(\mathrm{CP})$, registrado pela plataforma de força, é correlato ao $\mathrm{CG}$, sendo definido como o lugar da aplicação da resultante de todas as forças gravitacionais que agem no corpo ${ }^{(1,2,7)}$.

$\mathrm{O}$ uso da posturografia como ferramenta pode auxiliar, entre outros aspectos, o diagnóstico diferencial, a verificação de alterações no sistema vestíbulo-espinal, a avaliação do benefício de atividade física no controle postural, a avaliação da redução do controle postural com o envelhecimento e a criação de estratégias de prevenção a quedas de idosos ${ }^{(1,4,7,10,11)}$.

Atualmente, existem diversas plataformas de força sendo utilizadas para avaliar o equilíbrio corporal. No entanto, são equipamentos fabricados em outros países, de difícil acesso, dificultando seu uso no dia a dia clínico em nosso país ${ }^{(3-5,7-20)}$. Há também uma plataforma fixa fabricada no Brasil, mas sem testes de integração sensorial ou análise sensorial ${ }^{(5,19)}$, pois foi criada para uso nas áreas de biomecânica e ergonomia.

O posturógrafo Horus foi criado para uso específico em diagnóstico e reabilitação do equilíbrio, com engenharia e fabricação brasileiras. É um modelo de plataforma fixa inovador, por ter sido criado a partir da demanda de profissionais da área, com foco em portabilidade e baixo custo; inclui software desenvolvido com foco na usabilidade e baixo custo de manutenção, e a empresa oferece assistência técnica e calibragem no território nacional, garantindo medições corretas durante a vida útil do equipamento. Como diferencial, ajuda também a reabilitar os pacientes pela execução assistida de exercícios posturais usando a tecnologia de jogos por computador. Por se tratar de um equipamento lançado recentemente, em 2017, os valores de referência para indivíduos normais ainda não haviam sido estabelecidos, e o próprio protocolo para realização das provas necessárias para chegar ao diagnóstico postural não era claro. Por essa razão, este trabalho vem determinar uma forma de realizar as provas de forma expedita, além de investigar os valores de referência para diversas faixas etárias de voluntários hígidos de ambos os gêneros.

\section{OBJETIVO}

Propor um protocolo de investigação do equilíbrio corporal e determinar valores de referência para diferentes faixas etárias e gênero num sistema de posturografia estática com provas dinâmicas.

\section{MÉTODO}

Estudo analítico descritivo aplicado a uma amostra de 297 voluntários hígidos com idade entre 20 a 89 anos em 2018. O projeto foi enviado ao Comitê de ética da Irmandade da Santa Casa de Misericórdia de São Paulo, CAAE:88284318.1.0000.5479 e aprovado sob o número n. 2.713.595.

Antes de iniciar a pesquisa, todos os voluntários foram informados sobre o teor da pesquisa e os que aceitaram participar assinaram o termo de consentimento livre e esclarecido.

Os dados apresentados aqui foram levantados por vários profissionais especializados na área da otoneurologia, em diferentes serviços localizados nas cidades de Goiânia, Rio de Janeiro, Santa Maria e São Paulo

A posturografia estática com provas dinâmicas foi realizada com equipamento Horus, da marca Contronic. O conjunto pode ser visto na Figura 1. O posturógrafo é composto por uma

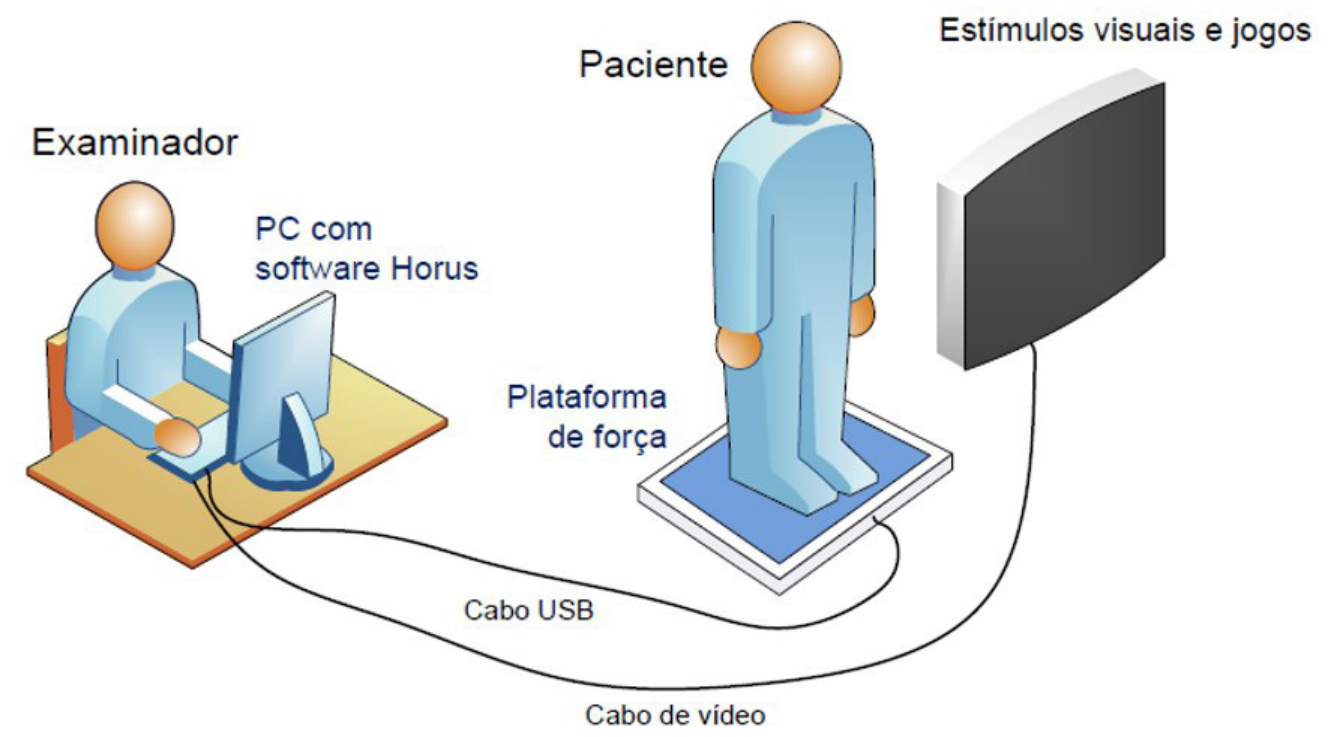

Figura 1. Diagrama funcional do sistema da plataforma estática com provas dinâmicas Horus 
plataforma de força conectada a um computador por um cabo USB que provê a comunicação digital e a alimentação elétrica da máquina. Os circuitos internos da plataforma têm as seguintes seções: quatro sensores de força do tipo strain gauge, circuito independente de condicionamento do sinal de cada sensor, quatro conversores de analógico para digital com resolução de 24 bits e microprocessador ARM de 32 bits com circuitos digitais associados. O software associado requer um computador com sistema operacional Windows. Sua função é exibir e registrar os dados fornecidos pela plataforma. Além da plataforma de força e do software, o sistema é acompanhado por uma almofada desenvolvida em material TPE (elastômero termoplástico), com altura de $5 \mathrm{~cm}$ e tamanho idêntico ao da etiqueta indelével existente na face superior da plataforma, contendo as mesmas marcas de referência para posicionamento dos pés.

Os estímulos visuais são gerados pelo mesmo software e foram projetados com auxílio de um televisor de 32 polegadas ou de um projetor, de acordo com o diagrama funcional (Figura 1).

\section{Seleção da amostra}

Os voluntários podiam participar da pesquisa de acordo com os seguintes critérios de exclusão: diagnóstico de doença audiovestibular considerada a autoavaliação do indivíduo: não escuta bem, queixa-se de tontura; apresenta doença neurológica, inclusive cinetose e enxaqueca; apresenta sintomas como desequilíbrio, instabilidade, visão turva, cabeça oca ou vertigem; reporta alterações que causem dores nos membros inferiores ou comprometam a força e a mobilidade; tem altura inferior a 1 metro ou peso acima de $130 \mathrm{~kg}$, tem déficit visual sem compensação por óculos ou lentes; toma alguma medicação antivertiginosa, antieméticos, para cefaleia, ansiolíticos ou antidepressivos; ingeriu cafeína ou álcool 48 horas antes da realização do procedimento.

Após o processo de elegibilidade, os voluntários calçaram as próprias meias ou o pró-pé oferecido pelos avaliadores.

\section{Estabilograma}

As oscilações posturais foram registradas para a esquerda $\mathrm{e}$ a direita pelo estabilograma ML (médio-lateral) e para a frente e para trás pelo estabilograma AP (anteroposterior). Ambos evidenciaram a amplitude de oscilação postural durante todo o tempo da prova, o que permitiu uma análise detalhada das amplitudes de oscilação corporal em qualquer momento.

Os estabilogramas permitiram a identificação visual dos períodos do teste nos quais o indivíduo apresentou maiores picos de oscilação, implicando maior risco de queda.

\section{Estatocinesigrama}

É o mapa do deslocamento do $\mathrm{CP}$ no eixo $\mathrm{ML}$ em relação ao deslocamento do CP no eixo AP. O CP do paciente variou no decorrer da prova, e cada medida gerou um ponto na coordenada atual, resultando numa "nuvem de pontos". Na prova de limite de estabilidade (LE), os pontos extremos em cada direção definiram a elipse de estabilidade; nas demais provas, a elipse de confiança foi calculada para abranger pelo menos $95 \%$ dos pontos que compunham a nuvem.

\section{Gráfico do espectro de frequências}

Para que o gráfico de frequência tenha um resolução adequada, o sinal no estabilograma deve durar pelo menos 30 segundos. Sinais mais curtos teriam menos amostras, e a resolução em frequência seria menor. Esse requisito foi introduzido pelo uso da FFT (fast fourier transform) para converter os sinais do domínio do tempo para o domínio da frequência, evitando técnicas que introduzem artefatos, como a de zero-padding.

A resolução da FFT foi expressa pelo número de barras verticais exibidas nos gráficos do domínio da frequência, conhecidas como bins. Se o deslocamento do $\mathrm{CP}$ for pequeno durante a prova, o tamanho dos bins no gráfico poderá ser pequeno. A altura de um bin, dada em milímetros, corresponde à intensidade do sinal naquela frequência específica, dada em Hertz.

A avaliação das bandas de frequência (frequency band, FB) é útil como medida quantitativa da velocidade com que variou a posição do $\mathrm{CP}$ ao longo da prova. Predominância de frequências baixas indica que o paciente fez poucas manobras de correção postural ao longo da prova ou que essas manobras foram lentas e/ou com baixa intensidade/força.

\section{Parâmetros quantitativos medidos em frequência}

FB0 indica a frequência abaixo da qual estão contidos 70,7\% da potência total do sinal. FB1 indica a frequência abaixo da qual estão contidos $80 \%$ da potência total do sinal. FB2 indica a frequência abaixo da qual estão contidos $85 \%$ da potência total do sinal. FB3 indica a frequência abaixo da qual estão contidos $90 \%$ da potência total do sinal. FB4 indica a frequência abaixo da qual estão contidos $95 \%$ da potência total do sinal. Estudos anteriores mostraram que FB1, com $80 \%$ da potência espectral, é o parâmetro que melhor caracteriza as alterações do sistema de controle postural ${ }^{(10)}$, razão pela qual foi adotado neste trabalho.

\section{Parâmetros quantitativos}

O parâmetro velocidade média, extraído a partir dos estabilogramas e dado em $\mathrm{mm} / \mathrm{s}$, indica a velocidade média do CP. De modo geral, quanto menor for a velocidade média do $\mathrm{CP}$, melhor será o equilíbrio corporal do paciente.

A partir do estatocinesigrama registrado em cada prova, foi calculada a elipse de confiança (EC), que abrange pelo menos $95 \%$ dos pontos medidos pela plataforma. Sua área fornece um parâmetro quantitativo do grau de desequilíbrio ou deslocamento do CP. O valor da área, em $\mathrm{mm}^{2}$, é fornecido no parâmetro área elipse de confiança 95\% (EC) do software Horus.

A relação percentual entre a $\mathrm{EC}$ em cada prova e a área do limite de estabilidade é denominada razão EC/LE. Quanto maior for a $\mathrm{EC}$, ou seja, quanto maior o deslocamento do $\mathrm{CP}$ durante a prova, maior será a razão EC/LE em porcentagem.

\section{Protocolo do exame}

\section{Orientação para o posicionamento dos pés na plataforma}

Os pacientes foram instruídos a posicionar os pés com os maléolos alinhados na linha tracejada horizontal da plataforma 
e mantê-los simetricamente afastados na linha anteroposterior. Os participantes até 59 anos ajustaram a posição do hálux apontando entre 0 e 15 graus. Os de 60 anos ou mais ajustaram o ângulo de cada pé entre 0 e 20 graus, na posição mais confortável. Os voluntários foram orientados a permanecer em "posição de sentido" durante todo o exame, sem dobrar o quadril e usando apenas a estratégia de tornozelo para manter o equilíbrio.

\section{Testes executados}

Teste de integração sensorial: os voluntários foram orientados a permanecer sobre a plataforma em posição ortostática durante 30 segundos, sem se distrair com perguntas ou conversas. Durante todo o tempo de execução, cada indivíduo permaneceu em silêncio, com os pés na mesma posição adotada no teste de limite de estabilidade. A plataforma e, portanto, os voluntários foram posicionados a um metro de distância da imagem gerada para prover estímulo visual, fosse por TV ou por projetor. O teste considerou as seguintes condições sensoriais:

- Condição 1 (C1): permanecer na posição ereta, com os olhos abertos, com os pés diretamente sobre a plataforma e sem o uso da almofada, olhando para um ponto fixo no centro da imagem com tamanho de $10 \%$. Esse percentual é o valor ajustado no software durante a programação do estímulo visual e define o tamanho do ponto visível na imagem contra um fundo preto;

- Condição 2 (C2): permanecer na posição ereta, com os olhos fechados, diretamente sobre a plataforma, isto é, sem o uso da almofada;

- Condição 3 (C3): permanecer na posição ereta em cima da almofada sobre a plataforma, com os olhos abertos, olhando para um ponto fixo com tamanho de $10 \%$;

- Condição 4 (C4): permanecer na posição ereta em cima da almofada sobre a plataforma, com os olhos fechados;

- Condição 5 (C5): permanecer na posição ereta em cima da almofada sobre a plataforma, olhando para uma imagem dinâmica que exibe barras que causam efeito optocinético, movendo-se para a direita com velocidade ajustada no software para $16 \%$;

- Condição 6 (C6): permanecer na posição ereta em cima da almofada sobre a plataforma, olhando para uma imagem dinâmica que exibe barras que causam efeito optocinético, movendo-se para a esquerda com velocidade ajustada no software para $16 \%$;

- Condição 7(C7): permanecer na posição ereta em cima da almofada sobre a plataforma, olhando para uma imagem dinâmica que exibe um túnel composto por barras finas, com direção para a frente e velocidade de $4 \%$ sem rotação, sendo todos esses parâmetros ajustados no software, na seção que define o estímulo visual.

Após a execução da $\mathrm{C} 2$, se os valores da elipse fossem menores que os da $\mathrm{C} 1$, esta condição foi repetida.

Analisaram-se os seguintes parâmetros quantitativos:
- área do limite de estabilidade, em $\mathrm{mm}^{2}$;

- teste de integração sensorial composto pela área da elipse de confiança (EC) em $\mathrm{mm}^{2}$, pela velocidade média AP em $\mathrm{mm} / \mathrm{s}$, pela velocidade média $\mathrm{ML}$ em $\mathrm{mm} / \mathrm{s}$, pela banda de frequência FB1 (80\%) AP em Hz e pela banda de frequência FB1 (80\%) ML em Hz, pelo equilíbrio funcional residual (EFR) em \% e pela análise sensorial (AS) em \%.

Os valores de EFR nas condições descritas foram calculados pelas equações de 1 a 7 .

A partir dos EFR determinados nas sete condições, foram calculados os parâmetros da análise sensorial pelas Equações 8 a 14: somatossensorial (SOM), visual (VIS), vestibular (VEST), dependência visual direita (DPVD), dependência visual esquerda (DPVE), dependência visual túnel (DPVT) e índice de equilíbrio composto (IEC).

$E F R \% C 1=100-$ Area ECC1 Área $L E * 100$

$E F R \% C 2=100-$ Área ECC2 Área $L E * 100$

$E F R \% C 3=100-$ Área ECC3 Área $L E * 100$

EFR\% C4 $=100-$ Area ECC4 Área LE*100

EFR\% C5 $=100-$ Área ECC5 Área $L E * 100$

EFR\% C6 $=100-$ Área ECC6 Área $L E * 100$

$E F R \% C 7=100-$ Área ECC7 Área $L E * 100$

$S O M \%=E F R \% C 2 E F R \% C 1 * 100$

$V I S \%=E F R \% C 3 E F R \% C 1 * 100$

$V E S T \%=E F R \% C 4 E F R \% C 1 * 100$

$D P V D \%=E F R \% C 5 E F R \% C 4 * 100$

$D P V E \%=E F R \% C 6 E F R \% C 4 * 100$

$D P V T \%=E F R \% C 7 E F R \% C 4 * 100$

$I E$ composto $\%=\left[\begin{array}{l}(E F R \% C 1+E F R \% C 2+3) *(E F R \% C 3+3) *(E F R \% C 4+3) * \\ (E F R \% C 5+3) *(E F R \% C 6+3) *(E F R \% C 7)\end{array}\right] / 17$

\section{Análise estatística}

Os resultados foram submetidos a análise estatística. Idade e gênero foram comparados com auxílio do teste de KruskalWallis. O nível de significância adotado foi de 5\% $(\mathrm{p}=0,05)$. A confiança estatística foi fixada em $95 \%$. 


\section{RESULTADOS}

Participaram do presente estudo 297 voluntários, sendo $172(57,9 \%)$ do gênero feminino e $125(42,1 \%)$ do gênero masculino. Foram divididos em seis grupos, sendo três do gênero feminino e três do gênero masculino, devido à diferença significativa $(p<0,01)$ na análise das comparações dos parâmetros da posturografia entre as faixas etárias e gênero, conforme o teste de Kruskal-Wallis (Tabela 1 e Tabela 2).

Nos valores de referência do limite de estabilidade, consideraram-se normais os valores percentis de $5 \%$, valores maiores ou iguais a $12.594 \mathrm{~mm}^{2}$ para a faixa de 20 a 59 anos no gênero feminino e maiores ou iguais a $19.221 \mathrm{~mm}^{2}$ no masculino. Na faixa de 60 a 69 anos, $7.031 \mathrm{~mm}^{2}$ no gênero

Tabela 1. Comparação das variáveis numéricas entre as idades

\begin{tabular}{|c|c|c|c|c|c|c|c|c|c|c|}
\hline IDADE & VARIÁVEL & $\mathrm{N}$ & MÉDIA & D.P & MíN & Q1 & MEDIANA & Q3 & MÁX & VALOR-P* \\
\hline \multirow[t]{19}{*}{$20-59(A)$} & LE & 141 & 24608.00 & 6247.00 & 8488.50 & 20286.00 & 24637.00 & 28603.00 & 42629.00 & $\begin{array}{c}P<0.001->A \neq B, \\
A \neq C, B \neq C\end{array}$ \\
\hline & $\mathrm{C} 1$ & 141 & 91.77 & 65.23 & 8.90 & 48.60 & 71.10 & 71.10 & 71.10 & $\begin{array}{c}P<0.001->A \neq B, \\
A \neq C, B \neq C\end{array}$ \\
\hline & EFR1 & 141 & 99.58 & 0.37 & 97.89 & 99.44 & 99.69 & 99.83 & 99.96 & $\begin{array}{c}P<0.001->A \neq B, \\
A \neq C, B \neq C\end{array}$ \\
\hline & $\mathrm{C} 2$ & 141 & 136.64 & 91.78 & 17.10 & 71.60 & 119.30 & 181.80 & 447.70 & $\begin{array}{c}\mathrm{P}<0.001-> \\
\mathrm{A} \neq(\mathrm{B}, \mathrm{C})\end{array}$ \\
\hline & $\mathrm{C} 3$ & 141 & 475.12 & 296.19 & 92.70 & 239.80 & 404.70 & 637.00 & 1473.50 & $\begin{array}{c}P<0.001->A \neq B, \\
A \neq C, B \neq C\end{array}$ \\
\hline & EFR3 & 141 & 97.88 & 1.62 & 92.11 & 97.49 & 98.50 & 98.96 & 99.59 & $\begin{array}{c}P<0.001->A \neq B, \\
A \neq C, B \neq C\end{array}$ \\
\hline & C5 & 141 & 789.05 & 392.17 & 253.20 & 525.40 & 696.80 & 981.60 & 2211.70 & $\begin{array}{c}P<0.001->A \neq B, \\
A \neq C, B \neq C\end{array}$ \\
\hline & EFR5 & 141 & 96.46 & 2.60 & 79.52 & 95.6 & 97.20 & 98.00 & 99.12 & $\begin{array}{c}P<0.001->A \neq B, \\
A \neq C, B \neq C\end{array}$ \\
\hline & C6 & 141 & 818.98 & 451.11 & 194.60 & 488.90 & 704.70 & 1042.80 & 2244.10 & $\begin{array}{c}P<0.001-> \\
A \neq(B, C)\end{array}$ \\
\hline & EFR6 & 141 & 96.34 & 2.70 & 81.24 & 95.66 & 97.22 & 98.04 & 99.07 & $\begin{array}{c}P<0.001->A \neq B, \\
A \neq C, B \neq C\end{array}$ \\
\hline & $\mathrm{C} 7$ & 141 & 763.65 & 406.01 & 191.10 & 452.10 & 662.30 & 954.10 & 2316.60 & $\begin{array}{c}P<0.001->A \neq B, \\
A \neq C, B \neq C\end{array}$ \\
\hline & EFR7 & 141 & 96.63 & 2.35 & 83.01 & 95.77 & 97.23 & 98.14 & 99.40 & $\begin{array}{c}P<0.001->A \neq B, \\
A \neq C, B \neq C\end{array}$ \\
\hline & SOM & 141 & 96.63 & 0.65 & 98.37 & 99.71 & 99.88 & 99.96 & 106.67 & $\begin{array}{c}P<0.001->A \neq B, \\
A \neq C, B \neq C\end{array}$ \\
\hline & VIS & 141 & 98.29 & 1.44 & 93.05 & 97.90 & 98.85 & 99.25 & 100.17 & $\begin{array}{c}P<0.001->A \neq B, \\
A \neq C, B \neq C\end{array}$ \\
\hline & VEST & 141 & 95.03 & 3.37 & 83.01 & 93.87 & 96.13 & 97.23 & 99.04 & $\begin{array}{c}P<0.001->A \neq B, \\
A \neq C, B \neq C\end{array}$ \\
\hline & DPVD & 141 & 102.00 & 2.73 & 95.45 & 100.32 & 101.49 & 102.78 & 112.57 & $\begin{array}{c}P<0.001-> \\
A \neq(B, C)\end{array}$ \\
\hline & DPVE & 141 & 101.87 & 2.78 & 94.47 & 100.35 & 101.44 & 102.52 & 114.01 & $\begin{array}{c}P<0.001->A \neq B, \\
A \neq C, B \neq C\end{array}$ \\
\hline & DPVT & 141 & 102.20 & 3.05 & 96.53 & 100.43 & 101.53 & 103.00 & 115.28 & $\begin{array}{c}P<0.001-> \\
A \neq(B, C)\end{array}$ \\
\hline & IEC & 141 & 96.75 & 2.07 & 86.47 & 96.07 & 97.34 & 98.19 & 99.06 & $\begin{array}{c}P<0.001->A \neq B, \\
A \neq C, B \neq C\end{array}$ \\
\hline \multirow[t]{2}{*}{$60-69(\mathrm{~A})$} & LE & 82 & 19097.00 & 7018.40 & 5870.40 & 14041.00 & 19160.00 & 23649.00 & 37601.00 & \\
\hline & C1 & 82 & 112.94 & 67.00 & 12.30 & 65.90 & 96.80 & 144.10 & 345.70 & \\
\hline
\end{tabular}

Legenda: N: voluntários. $\mathrm{P}^{\star}$ : Valor-P referente ao teste de Kruskal-Wallis para comparação dos valores entre 3 grupos. LE: limite de estabilidade(mm²). C1: condição olhos abertos superfície estável $\left(\mathrm{mm}^{2}\right)$. C2: condição olhos fechados superfície estável( $\left.\mathrm{mm}^{2}\right)$. C3: área elipse de confiança da condição olhos abertos superfície instável $\left(\mathrm{mm}^{2}\right)$. C4: condição olhos fechados superfície instável $\left(\mathrm{mm}^{2}\right)$. C5: condição estimulação optocinética para a direita superfície instável(mm²). C6: condição estimulação optocinética para a esquerda superfície instável( $\left.\mathrm{mm}^{2}\right)$. C7: condição estimulação túnel superfície instável(mm²). EFR1: olhos abertos, superfície estável(\%). EFR2: olhos fechados, superfície estável(\%). EFR3: olhos abertos, superfície instável(\%). EFR4: olhos fechados, superfície instável(\%)(\%). EFR5: estimulação optocinética para a direita, superfície instável. EFR6: estimulação optocinética para a esquerda, superfície instável(\%). EFR7: estimulação túnel, superfície instável(\%). SOM: somatossensorial(\%). VIS: visual(\%). VEST: vestibular(\%). DPVD: dependência visual optocinético para direita(\%). DPVE: dependência visual optocinético para esquerda(\%). DPVT: dependência visual túnel(\%). IEC: índice de equilíbrio composto(\%). 
Tabela 1. Continuação...

\begin{tabular}{|c|c|c|c|c|c|c|c|c|c|c|}
\hline IDADE & VARIÁVEL & $\mathrm{N}$ & MÉDIA & D.P & MÍN & Q1 & MEDIANA & Q3 & MÁX & VALOR-P* \\
\hline & EFR1 & 82 & 99.34 & 0.45 & 97.86 & 99.13 & 99.43 & 99.68 & 99.92 & \\
\hline & $\mathrm{C} 2$ & 82 & 210.92 & 129.09 & 35.00 & 120.10 & 177.15 & 258.50 & 625.40 & \\
\hline & EFR2 & 82 & 98.77 & 0.93 & 94.53 & 98.48 & 99.04 & 99.33 & 99.71 & \\
\hline & C3 & 82 & 600.26 & 302.05 & 107.40 & 371.10 & 574.75 & 752.10 & 1948.20 & \\
\hline & EFR3 & 82 & 96.40 & 2.31 & 89.12 & 95.67 & 97.10 & 98.06 & 99.38 & \\
\hline & $\mathrm{C} 4$ & 82 & 1786.20 & 908.52 & 478.20 & 1183.10 & 1530.30 & 2112.60 & 4810.30 & \\
\hline & EFR5 & 82 & 93.27 & 3.63 & 78.80 & 91.20 & 93.86 & 96.00 & 97.96 & \\
\hline & C6 & 82 & 1249.00 & 615.37 & 458.50 & 834.80 & 1044.90 & 1451.70 & 3213.20 & \\
\hline & EFR6 & 82 & 92.56 & 4.38 & 73.86 & 90.43 & 93.79 & 95.46 & 98.59 & \\
\hline & C7 & 82 & 1122.00 & 532.16 & 360.30 & 717.80 & 1019.50 & 1354.40 & 2544.70 & \\
\hline & EFR7 & 82 & 93.46 & 3.58 & 81.95 & 91.36 & 94.24 & 96.35 & 98.29 & \\
\hline & SOM & 82 & 99.42 & 0.74 & 95.75 & 99.26 & 99.62 & 99.77 & 100.41 & \\
\hline & VIS & 82 & 97.03 & 2.14 & 90.88 & 96.28 & 97.59 & 98.44 & 100.09 & \\
\hline & VEST & 82 & 90.11 & 6.09 & 67.39 & 87.54 & 91.70 & 93.85 & 99.25 & \\
\hline & DPVD & 82 & 104.51 & 5.75 & 93.78 & 101.37 & 103.28 & 105.94 & 132.21 & \\
\hline & DPVE & 82 & 103.68 & 5.90 & 93.41 & 100.57 & 102.11 & 104.94 & 132.40 & \\
\hline & DPVT & 82 & 104.73 & 5.97 & 94.26 & 101.60 & 103.63 & 106.66 & 134.05 & \\
\hline & IEC & 82 & 93.75 & 3.15 & 83.35 & 92.26 & 94.52 & 96.14 & 98.22 & \\
\hline \multirow[t]{22}{*}{$70-89(\mathrm{C})$} & LE & 74 & 14738.00 & 5463.70 & 5291.50 & 10346.00 & 14453.00 & 19195.00 & 26310.00 & \\
\hline & $\mathrm{C} 1$ & 74 & 152.81 & 84.42 & 18.40 & 84.40 & 136.90 & 213.10 & 386.50 & \\
\hline & EFR1 & 74 & 98.83 & 0.70 & 97.14 & 98.36 & 98.96 & 99.43 & 99.91 & \\
\hline & $\mathrm{C} 2$ & 74 & 265.04 & 162.46 & 19.50 & 135.50 & 227.60 & 366.20 & 699.90 & \\
\hline & EFR2 & 74 & 97.78 & 1.41 & 91.97 & 97.20 & 97.93 & 98.84 & 99.90 & \\
\hline & C3 & 74 & 823.80 & 415.73 & 208.50 & 502.40 & 717.70 & 1101.6 & 1940.7 & \\
\hline & EFR3 & 74 & 93.81 & 3.57 & 78.52 & 92.09 & 94.26 & 96.25 & 98.93 & \\
\hline & $\mathrm{C} 4$ & 74 & 2136.20 & 934.07 & 117.80 & 1581.50 & 2105.90 & 2629.60 & 5845.60 & \\
\hline & EFR4 & 74 & 83.86 & 8.49 & 54.82 & 78.05 & 84.89 & 90.73 & 97.81 & \\
\hline & C5 & 74 & 1319.60 & 540.07 & 467.00 & 917.90 & 1240.30 & 1607.80 & 3006.10 & \\
\hline & EFR5 & 74 & 89.98 & 5.12 & 73.72 & 88.00 & 90.56 & 93.63 & 97.41 & \\
\hline & C6 & 74 & 1410.40 & 681.35 & 354.70 & 895.20 & 1241.00 & 1744.60 & 3953.80 & \\
\hline & EFR6 & 74 & 89.15 & 6.46 & 71.35 & 86.78 & 90.75 & 93.23 & 97.79 & \\
\hline & $\mathrm{C} 7$ & 74 & 1388.30 & 535.60 & 474.40 & 969.00 & 1359.70 & 1723.00 & 2960.40 & \\
\hline & EFR7 & 74 & 89.23 & 5.97 & 64.32 & 86.76 & 90.23 & 93.27 & 97.37 & \\
\hline & SOM & 74 & 98.91 & 1.16 & 94.68 & 98.17 & 99.13 & 99.74 & 101.86 & \\
\hline & VIS & 74 & 94.89 & 3.38 & 79.71 & 93.24 & 95.64 & 97.39 & 99.03 & \\
\hline & VEST & 74 & 84.89 & 8.28 & 55.59 & 79.20 & 86.10 & 91.45 & 99.33 & \\
\hline & DPVD & 74 & 107.32 & 9.63 & 75.37 & 101.56 & 106.97 & 111.31 & 153.32 & \\
\hline & DPVE & 74 & 106.04 & 10.21 & 74.12 & 101.05 & 104.22 & 110.58 & 161.67 & \\
\hline & DPVT & 74 & 106.42 & 10.60 & 84.23 & 101.23 & 104.36 & 109.21 & 162.11 & \\
\hline & IEC & 74 & 90.27 & 4.43 & 76.89 & 88.59 & 90.73 & 93.29 & 97.77 & \\
\hline
\end{tabular}

Legenda: N: voluntários. $\mathrm{P}^{*}$ : Valor-P referente ao teste de Kruskal-Wallis para comparação dos valores entre 3 grupos. LE: limite de estabilidade(mm²). C1: condição olhos abertos superfície estável $\left(\mathrm{mm}^{2}\right)$. C2: condição olhos fechados superfície estável( $\left.\mathrm{mm}^{2}\right)$. C3: área elipse de confiança da condição olhos abertos superfície instável $\left(\mathrm{mm}^{2}\right)$. C4: condição olhos fechados superfície instável $\left(\mathrm{mm}^{2}\right)$. C5: condição estimulação optocinética para a direita superfície instável(mm²). C6: condição estimulação optocinética para a esquerda superfície instável( $\left.\mathrm{mm}^{2}\right)$. C7: condição estimulação túnel superfície instável(mm²). EFR1: olhos abertos, superfície estável(\%). EFR2: olhos fechados, superfície estável(\%). EFR3: olhos abertos, superfície instável(\%). EFR4: olhos fechados, superfície instável(\%)(\%). EFR5: estimulação optocinética para a direita, superfície instável. EFR6: estimulação optocinética para a esquerda, superfície instável(\%). EFR7: estimulação túnel, superfície instável(\%). SOM: somatossensorial(\%). VIS: visual(\%). VEST: vestibular(\%). DPVD: dependência visual optocinético para direita(\%). DPVE: dependência visual optocinético para esquerda(\%). DPVT: dependência visual túnel(\%). IEC: índice de equilíbrio composto(\%).

feminino e $12.161 \mathrm{~mm}^{2}$ no masculino; na faixa de 70 a 89 anos, $6.340 \mathrm{~mm}^{2}$ no gênero feminino e $8.794 \mathrm{~mm}^{2}$ no masculino.

Os valores percentis de $95 \%$ menores ou iguais nas condições C1, C2, C3, C4, C5, C6 e C7 em velocidade médio-lateral (VelML) e anteroposterior (VelAP) conforme faixas etárias e gênero (Tabela 3).
Valores de percentis dos parâmetros de posturografia no gênero feminino nas diferentes faixas etárias, considerando valores de referência P95 (Tabela 4).

Valores de percentis dos parâmetros de posturografia no gênero masculino nas diferentes faixas etárias, considerando valores de referência P95 (Tabela 5). 
Tabela 2. Comparação das variáveis numéricas entre gêneros

\begin{tabular}{|c|c|c|c|c|c|c|c|c|c|c|}
\hline GÊNERO & VARIÁVEL & $\mathrm{N}$ & MÉDIA & D.P & MíN & Q1 & MEDIANA & Q3 & MÁX & VALOR-P* \\
\hline \multirow[t]{37}{*}{ FEMININO } & IDADE & 172 & 56.90 & 17.80 & 20.00 & 43.00 & 62.00 & 71.00 & 87.00 & $P=0.031$ \\
\hline & LE & 172 & 18104.00 & 6864.20 & 5291.50 & 12507.00 & 18080.00 & 22608.00 & 37229.00 & $P<0.001$ \\
\hline & C1 & 172 & 111.11 & 76.80 & 8.90 & 57.35 & 89.45 & 140.15 & 386.50 & $P=0.419$ \\
\hline & VelMLC1 & 172 & 4.12 & 1.82 & 1.50 & 3.00 & 3.90 & 4.90 & 15.50 & $P=0.987$ \\
\hline & VelAPC1 & 172 & 6.81 & 2.33 & 2.00 & 5.20 & 6.25 & 7.90 & 16.20 & $P=0.023$ \\
\hline & EFR1 & 172 & 99.24 & 0.66 & 97.14 & 98.97 & 99.47 & 99.72 & 99.95 & $P=0.020$ \\
\hline & $\mathrm{C} 2$ & 172 & 180.01 & 135.52 & 17.10 & 83.05 & 149.45 & 216.75 & 699.90 & $P=0.064$ \\
\hline & VelMLC2 & 172 & 5.24 & 2.85 & 1.60 & 3.40 & 4.35 & 6.40 & 18.80 & $P=0.090$ \\
\hline & VelAPC2 & 172 & 10.52 & 4.78 & 4.00 & 7.10 & 9.40 & 12.60 & 31.90 & $P=0.018$ \\
\hline & EFR2 & 172 & 98.68 & 1.26 & 91.97 & 98.10 & 99.24 & 99.54 & 99.90 & $P=0.100$ \\
\hline & C3 & 172 & 559.64 & 342.05 & 92.70 & 293.50 & 491.15 & 725.20 & 1883.00 & $P=0.032$ \\
\hline & VelMLC3 & 172 & 10.68 & 3.88 & 3.20 & 8.10 & 10.00 & 12.80 & 25.60 & $P=0.014$ \\
\hline & VelAPC3 & 172 & 15.12 & 5.34 & 5.10 & 11.25 & 14.50 & 17.25 & 34.80 & $P=0.027$ \\
\hline & EFR3 & 172 & 96.18 & 2.97 & 83.73 & 94.39 & 97.06 & 98.55 & 99.59 & $P=0.075$ \\
\hline & C4 & 172 & 1504.80 & 855.57 & 117.80 & 861.95 & 1324.90 & 1965.80 & 4507.60 & $P=0.033$ \\
\hline & VelMLC4 & 172 & 18.90 & 7.36 & 5.10 & 13.75 & 17.70 & 23.10 & 43.50 & $P=0.006$ \\
\hline & VelAPC4 & 172 & 29.18 & 19.11 & 11.20 & 20.40 & 26.15 & 33.25 & 242.00 & $\mathrm{P}<0.001$ \\
\hline & EFR4 & 172 & 89.73 & 7.83 & 54.82 & 85.49 & 91.99 & 96.00 & 98.94 & $P=0.051$ \\
\hline & $\mathrm{C} 5$ & 172 & 935.91 & 457.61 & 260.30 & 583.25 & 834.40 & 1210.10 & 2394.50 & $P=0.005$ \\
\hline & VelMLC5 & 172 & 15.09 & 5.85 & 3.30 & 10.45 & 14.05 & 18.35 & 38.90 & $P=0.003$ \\
\hline & VelAPC5 & 172 & 21.66 & 7.47 & 9.10 & 16.55 & 19.95 & 25.35 & 52.90 & $P<0.001$ \\
\hline & EFR5 & 172 & 93.52 & 4.79 & 73.72 & 91.19 & 94.84 & 97.24 & 99.09 & $P=0.075$ \\
\hline & C6 & 172 & 1009.10 & 569.69 & 194.60 & 607.15 & 895.60 & 1245.50 & 3440.10 & $P=0.026$ \\
\hline & VelMLC6 & 172 & 14.41 & 5.58 & 4.80 & 10.45 & 13.80 & 17.15 & 46.50 & $P=0.003$ \\
\hline & VelAPC6 & 172 & 21.22 & 7.21 & 8.90 & 15.60 & 20.00 & 25.55 & 49.00 & $P<0.001$ \\
\hline & EFR6 & 172 & 93.04 & 5.46 & 72.49 & 90.75 & 94.47 & 96.93 & 99.06 & $P=0.072$ \\
\hline & $\mathrm{C7}$ & 172 & 963.48 & 519.60 & 191.10 & 575.35 & 855.90 & 1258.20 & 2960.40 & $P=0.052$ \\
\hline & VelMLC7 & 172 & 12.75 & 4.60 & 4.30 & 9.15 & 12.05 & 15.30 & 26.90 & $P=0.051$ \\
\hline & VelAPC7 & 172 & 22.20 & 8.73 & 8.30 & 15.90 & 20.50 & 26.60 & 69.00 & $P=0.067$ \\
\hline & EFR7 & 172 & 93.29 & 5.42 & 64.32 & 91.11 & 95.03 & 97.34 & 99.40 & $P=0.037$ \\
\hline & SOM & 172 & 99.47 & 1.05 & 94.68 & 99.23 & 99.73 & 99.92 & 106.67 & $P=0.821$ \\
\hline & VIS & 172 & 96.90 & 2.62 & 85.12 & 95.25 & 97.74 & 99.01 & 100.09 & $P=0.160$ \\
\hline & VEST & 172 & 90.41 & 7.49 & 55.59 & 86.70 & 92.31 & 96.23 & 99.33 & $P=0.060$ \\
\hline & DPVD & 172 & 104.41 & 7.16 & 75.37 & 100.85 & 102.46 & 106.47 & 153.32 & $P=0.160$ \\
\hline & DPVE & 172 & 103.77 & 7.57 & 74.12 & 100.38 & 101.97 & 105.54 & 161.67 & $P=0.372$ \\
\hline & DPVT & 172 & 104.13 & 7.54 & 84.23 & 100.58 & 102.40 & 106.28 & 162.11 & $P=0.732$ \\
\hline & IEC & 172 & 93.83 & 4.29 & 76.89 & 91.00 & 94.94 & 97.26 & 98.83 & $P=0.037$ \\
\hline \multirow[t]{9}{*}{ MASCULINO } & IDADE & 125 & 53.18 & 17.22 & 21.00 & 37.00 & 58.00 & 66.00 & 86.00 & \\
\hline & LE & 125 & 24100.00 & 6936.20 & 7020.60 & 20286.00 & 24080.00 & 29242.00 & 42629.00 & \\
\hline & $\mathrm{C} 1$ & 125 & 115.18 & 72.44 & 14.20 & 63.40 & 98.90 & 153.50 & 345.70 & \\
\hline & VelMLC1 & 125 & 4.14 & 1.72 & 1.90 & 2.90 & 3.70 & 5.00 & 13.20 & \\
\hline & VeLAPC1 & 125 & 7.27 & 2.13 & 4.00 & 5.70 & 6.70 & 8.60 & 16.30 & \\
\hline & EFR1 & 125 & 99.46 & 0.41 & 98.03 & 99.23 & 99.58 & 99.74 & 99.96 & \\
\hline & $\mathrm{C} 2$ & 125 & 201.70 & 131.22 & 19.00 & 99.50 & 161.10 & 265.40 & 625.40 & \\
\hline & VelMC2 & 125 & 5.70 & 2.98 & 2.10 & 3.70 & 4.80 & 6.80 & 17.90 & \\
\hline & VelAPC2 & 125 & 11.44 & 4.70 & 4.90 & 8.60 & 10.40 & 12.90 & 32.90 & \\
\hline
\end{tabular}

Legenda: N: voluntários. D.P: desvio padrão. $\mathrm{P}^{\star}$ :Valor-P referente ao teste de Mann-Whitney para comparação dos valores entre 2 grupos. LE: limite de estabilidade $\left(\mathrm{mm}^{2}\right)$. $\mathrm{C} 1$ : condição olhos abertos superfície estável( $\left.\mathrm{mm}^{2}\right)$. C2: condição olhos fechados superfície estável(mm²). C3: área elipse de confiança da condição olhos abertos superfície instável $\left(\mathrm{mm}^{2}\right)$. C4: condição olhos fechados superfície instável( $\left(\mathrm{mm}^{2}\right)$. C5: condição estimulação optocinética para a direita superfície instável( $\left.\mathrm{mm}^{2}\right)$. C6: condição estimulação optocinética para a esquerda superfície instável(mm²). C7: condição estimulação túnel superfície instável(mm²). VelML: velocidade médio-lateral(mm/s). VelAP: velocidade antero-posterior(mm/s). EFR1: olhos abertos, superfície estável(\%). EFR2: olhos fechados, superfície estável(\%). EFR3: olhos abertos, superfície instável(\%). EFR4: olhos fechados, superfície instável(\%)(\%). EFR5: estimulação optocinética para a direita, superfície instável. EFR6: estimulação optocinética para a esquerda, superfície instável(\%). EFR7: estimulação túnel, superfície instável(\%). SOM: somatossensorial(\%). VIS: visual(\%). VEST: vestibular(\%). DPVD: dependência visual optocinético para direita(\%). DPVE: dependência visual optocinético para esquerda(\%). DPVT: dependência visual túnel(\%). IEC: índice de equilíbrio composto(\%). 
Tabela 2. Continuação...

\begin{tabular}{|c|c|c|c|c|c|c|c|c|c|c|}
\hline GÊNERO & VARIÁVEL & $\mathrm{N}$ & MÉDIA & D.P & MÍN & Q1 & MEDIANA & Q3 & MÁX & VALOR-P* \\
\hline & EFR2 & 125 & 98.99 & 0.88 & 94.86 & 98.65 & 99.28 & 99.61 & 99.95 & \\
\hline & $\mathrm{C} 3$ & 125 & 647.33 & 377.04 & 150.00 & 365.70 & 548.20 & 813.40 & 1948.2 & \\
\hline & VelMLC3 & 125 & 11.99 & 4.65 & 4.20 & 8.70 & 11.40 & 14.90 & 34.00 & \\
\hline & VelAPC3 & 125 & 17.30 & 7.82 & 7.20 & 12.30 & 15.20 & 21.00 & 62.80 & \\
\hline & EFR3 & 125 & 96.84 & 2.84 & 78.52 & 96.03 & 97.72 & 98.65 & 99.46 & \\
\hline & $\mathrm{C} 4$ & 125 & 1730.50 & 944.26 & 410.70 & 1053.00 & 1589.70 & 2241.90 & 5845.60 & \\
\hline & VelMLC4 & 125 & 21.50 & 8.58 & 7.60 & 15.70 & 20.40 & 25.50 & 59.50 & \\
\hline & VelAPC4 & 125 & 33.04 & 14.45 & 12.90 & 23.30 & 30.50 & 38.70 & 132.70 & \\
\hline & EFR4 & 125 & 91.66 & 6.43 & 65.66 & 89.85 & 93.23 & 96.09 & 98.77 & \\
\hline & C5 & 125 & 1135.40 & 590.80 & 253.20 & 696.80 & 1026.90 & 1451.00 & 3159.20 & \\
\hline & VelMLC5 & 125 & 17.41 & 6.86 & 7.60 & 12.90 & 15.70 & 19.90 & 45.40 & \\
\hline & VelAPC5 & 125 & 25.84 & 9.71 & 11.50 & 18.80 & 23.40 & 31.70 & 62.70 & \\
\hline & EFRC5 & 125 & 94.58 & 4.02 & 77.36 & 93.63 & 95.85 & 97.29 & 99.12 & \\
\hline & $\mathrm{C} 6$ & 125 & 1189.60 & 667.32 & 207.90 & 693.50 & 966.30 & 1498.40 & 3953.80 & \\
\hline & VelMLC6 & 125 & 16.72 & 7.07 & 6.10 & 12.30 & 15.50 & 19.00 & 47.60 & \\
\hline & VelAPC6 & 125 & 25.02 & 9.79 & 9.80 & 19.10 & 22.90 & 28.70 & 60.10 & \\
\hline & EFR6 & 125 & 94.15 & 4.96 & 71.35 & 92.67 & 95.66 & 97.32 & 99.07 & \\
\hline & $\mathrm{C} 7$ & 125 & 1093.50 & 565.98 & 259.60 & 610.50 & 971.40 & 1390.80 & 2544.70 & \\
\hline & VelMLC7 & 125 & 14.05 & 5.66 & 1.40 & 9.80 & 13.00 & 16.90 & 36.20 & \\
\hline & VelAPC7 & 125 & 23.88 & 8.96 & 1.80 & 17.70 & 22.20 & 28.00 & 53.30 & \\
\hline & EFR7 & 125 & 94.76 & 3.93 & 80.08 & 93.77 & 96.00 & 97.46 & 99.03 & \\
\hline & SOM & 125 & 99.53 & 0.68 & 95.75 & 99.37 & 99.73 & 99.90 & 100.52 & \\
\hline & VIS & 125 & 97.36 & 2.64 & 79.71 & 96.61 & 98.23 & 98.92 & 100.17 & \\
\hline & VEST & 125 & 92.15 & 6.28 & 66.65 & 90.54 & 93.69 & 96.40 & 99.25 & \\
\hline & DPVD & 125 & 103.47 & 4.98 & 93.78 & 100.59 & 102.05 & 104.47 & 132.21 & \\
\hline & DPVE & 125 & 102.93 & 4.50 & 94.04 & 100.46 & 101.69 & 104.03 & 123.26 & \\
\hline & DPVT & 125 & 103.70 & 5.39 & 94.26 & 100.81 & 102.21 & 104.55 & 131.32 & \\
\hline & IEC & 125 & 94.97 & 3.67 & 77.77 & 93.70 & 96.06 & 97.56 & 99.06 & \\
\hline
\end{tabular}

Legenda: N: voluntários. D.P: desvio padrão. $\mathrm{P}^{\star}$ :Valor-P referente ao teste de Mann-Whitney para comparação dos valores entre 2 grupos. LE: limite de estabilidade $\left(\mathrm{mm}^{2}\right)$. C1: condição olhos abertos superfície estável $\left(\mathrm{mm}^{2}\right)$. C2: condição olhos fechados superfície estável(mm²). C3: área elipse de confiança da condição olhos abertos superfície instável $\left(\mathrm{mm}^{2}\right)$. C4: condição olhos fechados superfície instável(mm²). C5: condição estimulação optocinética para a direita superfície instável( $\left.\mathrm{mm}^{2}\right)$. C6: condição estimulação optocinética para a esquerda superfície instável(mm²). C7: condição estimulação túnel superfície instável(mm²). VelML: velocidade médio-lateral(mm/s). VelAP: velocidade antero-posterior(mm/s). EFR1: olhos abertos, superfície estável(\%). EFR2: olhos fechados, superfície estável(\%). EFR3: olhos abertos, superfície instável(\%). EFR4: olhos fechados, superfície instável(\%)(\%). EFR5: estimulação optocinética para a direita, superfície instável. EFR6: estimulação optocinética para a esquerda, superfície instável(\%). EFR7: estimulação túnel, superfície instável(\%). SOM: somatossensorial(\%). VIS: visual(\%). VEST: vestibular(\%). DPVD: dependência visual optocinético para direita(\%). DPVE: dependência visual optocinético para esquerda(\%). DPVT: dependência visual túnel(\%). IEC: índice de equilíbrio composto(\%).

Tabela 3. Valores de percentis da velocidade médio-lateral (VeIML) e anteroposterior (VelAP) conforme faixas etárias e gênero

\begin{tabular}{ccccccccccc}
\hline IDADE & VARIÁVEL & $\mathrm{N}$ & GÊNERO & $\mathrm{C} 1$ & $\mathrm{C} 2$ & $\mathrm{C} 3$ & $\mathrm{C} 4$ & $\mathrm{C} 5$ & $\mathrm{C}$ & $\mathrm{C}$ \\
\hline \multirow{2}{*}{$20-59$} & VelML & 75 & feminino & $<5,80$ & $<7,80$ & $<15,70$ & $<27,50$ & $<19,50$ & $<18,50$ & $<15,80$ \\
& VelML & 65 & masculino & $<6,30$ & $<8,50$ & $<16,20$ & $<28,80$ & $<22,80$ & $<19,50$ & $<17,50$ \\
\multirow{2}{*}{$60-69$} & VelML & 45 & feminino & $<5,50$ & $<10,40$ & $<15,70$ & $<33,30$ & $<28,10$ & $<23,10$ & $<21,30$ \\
& VelML & 37 & masculino & $<8,20$ & $<14,20$ & $<21,80$ & $<40,20$ & $<31,10$ & $<32,30$ & $<27,70$ \\
\multirow{2}{*}{$70-89$} & VelML & 51 & feminino & $<8,20$ & $<16,20$ & $<20,60$ & $<34,90$ & $<28,10$ & $<28,10$ & $<25,90$ \\
& VelML & 23 & masculino & $<8,30$ & $<14,70$ & $<22,70$ & $<40,20$ & $<33,70$ & $<37,00$ & $<28,90$ \\
\multirow{2}{*}{$20-59$} & VelAP & 75 & feminino & $<9,60$ & $<17,40$ & $<19,60$ & $<40,80$ & $<26,50$ & $<26,10$ & $<26,30$ \\
& VelAP & 65 & masculino & $<10,80$ & $<15,70$ & $<23,70$ & $<44,70$ & $<34,90$ & $<30,10$ & $<38,00$ \\
$60-69$ & VelAP & 45 & feminino & $<11,30$ & $<21,10$ & $<25,80$ & $<47,50$ & $<33,70$ & $<39,40$ & $<43,90$ \\
& VelAP & 37 & masculino & $<38,00$ & $<23,50$ & $<27,40$ & $<58,50$ & $<42,30$ & $<48,60$ & $<46,20$ \\
$70-89$ & VelAP & 51 & feminino & $<12,10$ & $<21,60$ & $<30,80$ & $<57,80$ & $<46,20$ & $<39,80$ & $<42,90$ \\
& VelAP & 23 & masculino & $<12,50$ & $<23,30$ & $<44,80$ & $<64,00$ & $<52,10$ & $<57,70$ & $<45,70$ \\
\hline
\end{tabular}

Legenda: N: voluntários. C1: condição olhos abertos superfície estável(mm²). C2: condição olhos fechados superfície estável(mm²). C3: área elipse de confiança da condição olhos abertos superfície instável $\left(\mathrm{mm}^{2}\right)$. C4: condição olhos fechados superfície instável(mm²). C5: condição estimulação optocinética para a direita superfície instável( $\left(\mathrm{mm}^{2}\right)$. C6: condição estimulação optocinética para a esquerda superfície instável(mm²). C7: condição estimulação túnel superfície instável(mm²). $\mathrm{N}$ : voluntários. 
Tabela 4. Valores de percentis dos parâmetros de posturografia para o gênero feminino nas diferentes faixas etárias

\begin{tabular}{|c|c|c|c|c|c|c|c|c|c|c|c|}
\hline IDADE & VARIÁVEL & $\mathrm{N}$ & $\mathrm{P} 1$ & P5 & $\mathrm{P} 10$ & P25 & $\mathrm{P} 50$ & P75 & $\mathrm{P90}$ & P95 & P99 \\
\hline \multirow[t]{22}{*}{$20-59$} & LE & 76 & 8488.50 & 12594.00 & 15117.00 & 18217.00 & 22162.00 & 26016.00 & 30723.00 & 32615.00 & 37229.00 \\
\hline & C1 & 76 & 8.90 & 18.10 & 26.80 & 46.30 & 67.20 & 109.45 & 155.10 & 203.90 & 345.20 \\
\hline & EFR1 & 76 & 97.89 & 98.42 & 99.06 & 99.44 & 99.69 & 99.82 & 99.90 & 99.92 & 99.95 \\
\hline & $\mathrm{C} 2$ & 76 & 17.10 & 30.20 & 38.60 & 63.25 & 110.20 & 169.70 & 215.00 & 313.70 & 414.50 \\
\hline & EFR2 & 76 & 97.44 & 98.08 & 98.48 & 99.25 & 99.49 & 99.74 & 99.85 & 99.88 & 99.90 \\
\hline & C3 & 76 & 92.70 & 164.00 & 184.40 & 211.85 & 328.10 & 626.75 & 865.20 & 964.10 & 1185.20 \\
\hline & EFR3 & 76 & 92.11 & 94.14 & 94.51 & 97.01 & 98.56 & 99.02 & 99.26 & 99.43 & 99.59 \\
\hline & C4 & 76 & 310.90 & 361.10 & 467.70 & 636.90 & 932.10 & 1301.60 & 1734.60 & 2496.50 & 3654.20 \\
\hline & EFR4 & 76 & 81.51 & 86.00 & 89.07 & 93.35 & 96.05 & 97.42 & 98.27 & 98.48 & 98.94 \\
\hline & C5 & 76 & 260.30 & 271.30 & 326.10 & 451.15 & 635.80 & 881.75 & 1171.10 & 1442.30 & 2165.00 \\
\hline & EFR5 & 76 & 79.52 & 92.06 & 92.94 & 95.52 & 97.27 & 98.17 & 98.68 & 98.94 & 99.09 \\
\hline & $\mathrm{C} 6$ & 76 & 194.60 & 284.10 & 345.70 & 404.65 & 619.40 & 893.25 & 1315.70 & 1592.10 & 1699.90 \\
\hline & EFR6 & 76 & 81.24 & 91.69 & 92.68 & 95.62 & 97.41 & 98.24 & 98.60 & 98.86 & 99.06 \\
\hline & C7 & 76 & 191.10 & 233.40 & 294.80 & 414.30 & 621.75 & 908.55 & 1056.70 & 1363.00 & 2316.60 \\
\hline & EFR7 & 76 & 83.01 & 91.43 & 94.35 & 96.05 & 97.40 & 98.27 & 98.70 & 98.81 & 99.40 \\
\hline & SOM & 76 & 98.37 & 99.20 & 99.55 & 99.66 & 99.88 & 99.96 & 100.07 & 100.23 & 106.67 \\
\hline & VIS & 76 & 93.63 & 94.72 & 95.28 & 97.67 & 98.99 & 99.28 & 99.43 & 99.72 & 99.83 \\
\hline & VEST & 76 & 83.01 & 87.25 & 89.64 & 93.83 & 96.40 & 97.73 & 98.46 & 98.79 & 99.04 \\
\hline & DPVD & 76 & 95.45 & 98.82 & 99.48 & 100.26 & 101.40 & 102.62 & 105.54 & 108.70 & 112.34 \\
\hline & DPVE & 76 & 94.47 & 98.99 & 99.31 & 100.16 & 101.43 & 102.66 & 105.74 & 108.48 & 111.56 \\
\hline & DPVT & 76 & 96.53 & 98.64 & 99.22 & 100.34 & 101.58 & 102.97 & 106.60 & 109.74 & 115.28 \\
\hline & IEC & 76 & 86.47 & 92.67 & 93.86 & 96.11 & 97.41 & 98.34 & 98.70 & 98.78 & 98.83 \\
\hline \multirow[t]{22}{*}{$60-69$} & LE & 45 & 5870.40 & 7031.00 & 8703.10 & 11875.00 & 14674.00 & 19762.00 & 23649.00 & 27114.00 & 31011.00 \\
\hline & C1 & 45 & 12.30 & 22.90 & 33.40 & 62.00 & 91.50 & 139.20 & 186.50 & 222.40 & 310.40 \\
\hline & EFR1 & 45 & 97.86 & 98.15 & 98.60 & 99.12 & 99.37 & 99.60 & 99.77 & 99.84 & 99.92 \\
\hline & $\mathrm{C} 2$ & 45 & 35.00 & 61.80 & 68.30 & 98.50 & 162.10 & 206.70 & 302.90 & 494.60 & 606.10 \\
\hline & EFR2 & 45 & 94.53 & 96.99 & 97.25 & 98.32 & 99.12 & 99.33 & 99.51 & 99.55 & 99.71 \\
\hline & C3 & 45 & 107.40 & 190.70 & 247.30 & 368.60 & 491.90 & 700.00 & 951.40 & 1011.90 & 1283.10 \\
\hline & EFR3 & 45 & 90.24 & 90.85 & 92.23 & 95.10 & 96.36 & 97.91 & 98.39 & 98.66 & 99.38 \\
\hline & C4 & 45 & 478.20 & 647.60 & 840.30 & 1183.10 & 1508.00 & 2112.60 & 2714.30 & 3312.80 & 4391.40 \\
\hline & EFR4 & 45 & 69.86 & 73.01 & 79.51 & 85.51 & 89.85 & 92.88 & 94.34 & 95.07 & 97.01 \\
\hline & C5 & 45 & 482.40 & 560.80 & 569.30 & 735.80 & 952.00 & 1234.10 & 1903.00 & 2132.40 & 2394.50 \\
\hline & EFR5 & 45 & 78.80 & 86.50 & 87.39 & 90.93 & 92.66 & 95.73 & 96.90 & 97.17 & 97.95 \\
\hline & $\mathrm{C} 6$ & 45 & 458.50 & 586.40 & 711.50 & 840.10 & 991.20 & 1372.20 & 2389.70 & 2629.10 & 3213.20 \\
\hline & EFR6 & 45 & 73.86 & 83.86 & 85.68 & 88.94 & 92.62 & 95.04 & 96.13 & 96.42 & 97.13 \\
\hline & $\mathrm{C7}$ & 45 & 360.30 & 482.10 & 509.50 & 717.80 & 931.70 & 1228.10 & 1620.60 & 1906.60 & 2147.50 \\
\hline & EFR7 & 45 & 81.95 & 85.84 & 88.43 & 91.13 & 93.20 & 95.93 & 96.57 & 96.89 & 97.46 \\
\hline & SOM & 45 & 96.51 & 97.90 & 98.37 & 99.25 & 99.65 & 99.78 & 99.98 & 100.04 & 100.35 \\
\hline & VIS & 45 & 91.36 & 91.45 & 92.55 & 95.54 & 97.22 & 98.46 & 99.25 & 99.95 & 100.09 \\
\hline & VEST & 45 & 70.43 & 74.05 & 79.80 & 87.10 & 90.48 & 93.45 & 94.72 & 95.65 & 97.16 \\
\hline & DPVD & 45 & 99.92 & 100.24 & 100.27 & 101.97 & 103.65 & 106.94 & 110.07 & 114.66 & 130.40 \\
\hline & DPVE & 45 & 93.41 & 97.87 & 98.67 & 100.49 & 102.77 & 105.72 & 110.53 & 111.87 & 132.40 \\
\hline & DPVT & 45 & 98.12 & 98.94 & 99.68 & 102.36 & 104.47 & 108.41 & 113.12 & 117.30 & 134.05 \\
\hline & IEC & 45 & 83.35 & 87.57 & 88.83 & 91.08 & 93.55 & 95.47 & 96.59 & 96.72 & 97.34 \\
\hline \multirow[t]{6}{*}{$70-89$} & LE & 51 & 5291.50 & 6340.20 & 8194.10 & 9644.30 & 12842.00 & 17132.00 & 20133.00 & 21262.00 & 23878.00 \\
\hline & $\mathrm{C} 1$ & 51 & 18.40 & 42.50 & 57.80 & 84.90 & 136.40 & 213.70 & 273.00 & 327.10 & 386.50 \\
\hline & EFR1 & 51 & 97.14 & 97.24 & 97.57 & 98.21 & 98.83 & 99.41 & 99.61 & 99.66 & 99.91 \\
\hline & $\mathrm{C} 2$ & 51 & 19.50 & 61.40 & 79.70 & 122.20 & 218.50 & 350.30 & 487.30 & 633.30 & 699.90 \\
\hline & EFR2 & 51 & 91.97 & 94.64 & 96.06 & 96.88 & 97.92 & 98.68 & 99.48 & 99.64 & 99.90 \\
\hline & C3 & 51 & 208.50 & 338.30 & 421.20 & 482.10 & 594.50 & 964.80 & 1350.10 & 1576.50 & 1883.00 \\
\hline
\end{tabular}

Legenda: N: voluntários. LE: limite de estabilidade $\left(\mathrm{mm}^{2}\right)$. C1: condição olhos abertos superfície estável(mm²). C2: condição olhos fechados superfície estável(mm²). C3: área elipse de confiança da condição olhos abertos superfície instável( $\left.\mathrm{mm}^{2}\right)$. C4: condição olhos fechados superfície instável(mm²). C5: condição estimulação optocinética para a direita superfície instável( $\left.\mathrm{mm}^{2}\right)$. C6: condição estimulação optocinética para a esquerda superfície instável(mm²). C7: condição estimulação túnel superfície instável( $\left.\mathrm{mm}^{2}\right)$. EFR1: olhos abertos, superfície estável(\%). EFR2: olhos fechados, superfície estável(\%). EFR3: olhos abertos, superfície instável(\%). EFR4: olhos fechados, superfície instável(\%)(\%). EFR5: estimulação optocinética para a direita, superfície instável. EFR6: estimulação optocinética para a esquerda, superfície instável(\%). EFR7: estimulação túnel, superfície instável(\%). SOM: somatossensorial(\%). VIS: visual(\%). VEST: vestibular(\%). DPVD: dependência visual optocinético para direita(\%). DPVE: dependência visual optocinético para esquerda(\%). DPVT: dependência visual túnel(\%). IEC: índice de equilíbrio composto(\%). 
Tabela 4. Continuação...

\begin{tabular}{|c|c|c|c|c|c|c|c|c|c|c|c|}
\hline IDADE & VARIÁVEL & $\mathrm{N}$ & $\mathrm{P} 1$ & P5 & P10 & P25 & P50 & P75 & P90 & P95 & P99 \\
\hline & EFR3 & 51 & 83.73 & 86.90 & 90.05 & 92.04 & 94.32 & 96.25 & 97.50 & 97.80 & 98.93 \\
\hline & $\mathrm{C} 4$ & 51 & 117.80 & 765.40 & 847.10 & 1196.50 & 1839.20 & 2535.30 & 2930.10 & 3501.80 & 4507.60 \\
\hline & EFR4 & 51 & 54.82 & 70.60 & 71.54 & 78.42 & 84.77 & 90.87 & 94.40 & 95.40 & 97.81 \\
\hline & C5 & 51 & 467.00 & 574.00 & 628.10 & 782.10 & 1177.70 & 1458.60 & 1680.60 & 2082.80 & 2160.40 \\
\hline & EFR5 & 51 & 73.72 & 79.18 & 84.04 & 88.00 & 90.59 & 93.31 & 95.90 & 96.73 & 97.41 \\
\hline & $\mathrm{C} 6$ & 51 & 354.70 & 519.50 & 698.70 & 889.10 & 1118.00 & 1504.30 & 1975.00 & 2234.80 & 3440.10 \\
\hline & EFR6 & 51 & 72.49 & 75.02 & 81.35 & 87.39 & 90.70 & 93.86 & 95.63 & 96.74 & 97.79 \\
\hline & $\mathrm{C} 7$ & 51 & 474.40 & 537.30 & 774.20 & 946.40 & 1309.80 & 1692.60 & 1959.50 & 2351.20 & 2960.40 \\
\hline & EFR7 & 51 & 64.32 & 73.95 & 82.38 & 86.42 & 89.70 & 92.30 & 95.05 & 96.32 & 97.37 \\
\hline & SOM & 51 & 94.68 & 96.29 & 97.44 & 98.16 & 99.07 & 99.74 & 100.00 & 100.41 & 101.86 \\
\hline & VIS & 51 & 85.12 & 89.46 & 91.84 & 93.18 & 95.26 & 97.63 & 98.01 & 98.77 & 99.03 \\
\hline & VEST & 51 & 55.59 & 71.46 & 72.99 & 80.06 & 85.69 & 91.71 & 94.85 & 95.79 & 99.33 \\
\hline & DPVD & 51 & 75.37 & 92.96 & 99.45 & 101.59 & 107.50 & 110.80 & 118.94 & 122.25 & 153.32 \\
\hline & DPVE & 51 & 74.12 & 91.23 & 98.28 & 101.31 & 104.46 & 108.71 & 116.20 & 122.24 & 161.67 \\
\hline & DPVT & 51 & 84.23 & 93.37 & 94.11 & 99.38 & 103.50 & 108.71 & 116.74 & 121.53 & 162.11 \\
\hline & IEC & 51 & 76.89 & 81.88 & 84.84 & 88.59 & 90.72 & 93.27 & 95.69 & 96.13 & 97.77 \\
\hline
\end{tabular}

Legenda: $\mathrm{N}$ : voluntários. LE: limite de estabilidade $\left(\mathrm{mm}^{2}\right)$. C1: condição olhos abertos superfície estável( $\left.\mathrm{mm}^{2}\right)$. C2: condição olhos fechados superfície estável(mm²). C3: área elipse de confiança da condição olhos abertos superfície instável( $\left(\mathrm{mm}^{2}\right)$. C4: condição olhos fechados superfície instável(mm²). C5: condição estimulação optocinética para a direita superfície instável $\left(\mathrm{mm}^{2}\right)$. C6: condição estimulação optocinética para a esquerda superfície instável(mm²). C7: condição estimulação túnel superfície instável( $\left.\mathrm{mm}^{2}\right)$. EFR1: olhos abertos, superfície estável(\%). EFR2: olhos fechados, superfície estável(\%). EFR3: olhos abertos, superfície instável(\%). EFR4: olhos fechados, superfície instável(\%)(\%). EFR5: estimulação optocinética para a direita, superfície instável. EFR6: estimulação optocinética para a esquerda, superfície instável(\%). EFR7: estimulação túnel, superfície instável(\%). SOM: somatossensorial(\%). VIS: visual(\%). VEST: vestibular(\%). DPVD: dependência visual optocinético para direita(\%). DPVE: dependência visual optocinético para esquerda(\%). DPVT: dependência visual túnel(\%). IEC: índice de equilíbrio composto(\%).

Tabela 5. Valores de percentis dos parâmetros de posturografia para gênero masculino nas diferentes faixas etárias

\begin{tabular}{|c|c|c|c|c|c|c|c|c|c|c|c|}
\hline IDADE & VARIÁVEL & $\mathrm{N}$ & P1 & P5 & P10 & P25 & P50 & P75 & P90 & P95 & P99 \\
\hline \multirow[t]{22}{*}{$20-59$} & LE & 65 & 17171.00 & 19221.00 & 20286.00 & 22606.00 & 26057.00 & 30583.00 & 35195.00 & 36529.00 & 42629.00 \\
\hline & $\mathrm{C} 1$ & 65 & 14.20 & 25.10 & 30.00 & 49.90 & 87.90 & 124.30 & 186.30 & 237.80 & 291.70 \\
\hline & EFR1 & 65 & 98.70 & 99.12 & 99.17 & 99.49 & 99.70 & 99.83 & 99.88 & 99.92 & 99.96 \\
\hline & $\mathrm{C} 2$ & 65 & 19.00 & 50.00 & 55.40 & 75.90 & 124.70 & 191.10 & 296.50 & 352.00 & 447.70 \\
\hline & EFR2 & 65 & 98.30 & 98.39 & 98.60 & 99.32 & 99.56 & 99.71 & 99.79 & 99.81 & 99.95 \\
\hline & C3 & 65 & 150.00 & 188.90 & 218.00 & 320.30 & 440.90 & 637.00 & 1050.20 & 1105.40 & 1473.50 \\
\hline & EFR3 & 65 & 92.34 & 95.54 & 95.97 & 97.61 & 98.47 & 98.85 & 99.09 & 99.35 & 99.46 \\
\hline & C4 & 65 & 410.70 & 502.20 & 601.30 & 827.60 & 1169.50 & 1805.60 & 2386.30 & 2787.80 & 3557.00 \\
\hline & EFR4 & 65 & 84.68 & 88.16 & 90.52 & 93.23 & 95.50 & 96.65 & 97.83 & 98.04 & 98.77 \\
\hline & C5 & 65 & 253.20 & 389.10 & 482.70 & 594.10 & 833.50 & 1060.20 & 1451.00 & 1787.20 & 2211.70 \\
\hline & EFR5 & 65 & 90.70 & 93.59 & 94.28 & 95.77 & 96.99 & 97.75 & 98.42 & 98.81 & 99.12 \\
\hline & C6 & 65 & 207.90 & 367.80 & 477.90 & 590.00 & 773.60 & 1212.10 & 1663.60 & 2113.00 & 2244.10 \\
\hline & EFR6 & 65 & 88.32 & 92.39 & 92.94 & 95.94 & 97.05 & 97.80 & 98.50 & 98.81 & 99.07 \\
\hline & $\mathrm{C} 7$ & 65 & 259.60 & 291.20 & 390.30 & 536.80 & 806.50 & 1252.00 & 1423.90 & 1647.30 & 1972.40 \\
\hline & EFR7 & 65 & 89.74 & 93.36 & 94.02 & 95.49 & 97.13 & 98.00 & 98.77 & 98.90 & 99.03 \\
\hline & SOM & 65 & 98.86 & 99.05 & 99.47 & 99.71 & 99.88 & 99.96 & 100.06 & 100.16 & 100.52 \\
\hline & VIS & 65 & 93.05 & 96.04 & 96.38 & 98.16 & 98.77 & 99.10 & 99.44 & 99.58 & 100.17 \\
\hline & VEST & 65 & 85.14 & 88.35 & 91.09 & 94.00 & 95.77 & 96.98 & 97.93 & 98.13 & 98.91 \\
\hline & DPVD & 65 & 98.48 & 99.05 & 99.88 & 100.35 & 101.73 & 103.05 & 104.65 & 107.11 & 112.57 \\
\hline & DPVE & 65 & 97.70 & 98.97 & 99.29 & 100.46 & 101.44 & 102.47 & 104.39 & 105.60 & 114.01 \\
\hline & DPVT & 65 & 98.15 & 99.08 & 99.99 & 100.76 & 101.43 & 103.25 & 105.42 & 107.32 & 113.28 \\
\hline & IEC & 65 & 90.29 & 93.79 & 94.92 & 95.89 & 97.29 & 97.86 & 98.37 & 98.53 & 99.06 \\
\hline \multirow[t]{2}{*}{$60-69$} & LE & 37 & 11744.00 & 12161.00 & 14455.00 & 19662.00 & 21392.00 & 27393.00 & 30469.00 & 31627.00 & 37601.00 \\
\hline & C1 & 37 & 40.90 & 44.00 & 53.40 & 69.20 & 100.50 & 154.00 & 192.00 & 301.20 & 345.70 \\
\hline
\end{tabular}

Legenda: N: voluntários.LE: limite de estabilidade $\left(\mathrm{mm}^{2}\right)$. C1: condição olhos abertos superfície estável(mm²). C2: condição olhos fechados superfície estável(mm²). C3: área elipse de confiança da condição olhos abertos superfície instável(mm²). C4: condição olhos fechados superfície instável(mm²). C5: condição estimulação optocinética para a direita superfície instável $\left(\mathrm{mm}^{2}\right)$. C6: condição estimulação optocinética para a esquerda superfície instável(mm²). C7: condição estimulação túnel superfície instável( $\left.\mathrm{mm}^{2}\right)$. EFR1: olhos abertos, superfície estável(\%). EFR2: olhos fechados, superfície estável(\%). EFR3: olhos abertos, superfície instável(\%). EFR4: olhos fechados, superfície instável(\%)(\%). EFR5: estimulação optocinética para a direita, superfície instável. EFR6: estimulação optocinética para a esquerda, superfície instável(\%). EFR7: estimulação túnel, superfície instável(\%). SOM: somatossensorial(\%). VIS: visual(\%). VEST: vestibular(\%). DPVD: dependência visual optocinético para direita(\%). DPVE: dependência visual optocinético para esquerda(\%). DPVT: dependência visual túnel(\%). IEC: índice de equilíbrio composto(\%). 
Tabela 5. Continuação...

\begin{tabular}{|c|c|c|c|c|c|c|c|c|c|c|c|}
\hline IDADE & VARIÁVEL & $\mathrm{N}$ & $\mathrm{P} 1$ & P5 & P10 & P25 & P50 & P75 & P90 & P95 & P99 \\
\hline & EFR1 & 37 & 98.07 & 98.99 & 99.06 & 99.31 & 99.50 & 99.69 & 99.79 & 99.85 & 99.86 \\
\hline & $\mathrm{C} 2$ & 37 & 66.10 & 89.20 & 102.40 & 150.40 & 226.70 & 307.40 & 495.00 & 522.70 & 625.40 \\
\hline & EFR2 & 37 & 94.86 & 97.05 & 98.24 & 98.58 & 98.96 & 99.28 & 99.59 & 99.63 & 99.67 \\
\hline & C3 & 37 & 173.60 & 236.00 & 286.20 & 384.60 & 683.00 & 778.60 & 985.80 & 1204.90 & 1948.20 \\
\hline & EFR3 & 37 & 89.12 & 92.06 & 94.13 & 96.42 & 97.42 & 98.10 & 98.97 & 99.01 & 99.37 \\
\hline & C4 & 37 & 581.70 & 744.00 & 864.40 & 1188.90 & 1624.40 & 2061.00 & 3621.80 & 3941.60 & 4810.30 \\
\hline & EFR4 & 37 & 66.72 & 84.70 & 85.25 & 89.85 & 92.55 & 93.90 & 96.47 & 96.57 & 98.45 \\
\hline & C5 & 37 & 467.60 & 468.40 & 603.80 & 844.90 & 1171.70 & 1509.90 & 1867.50 & 2735.10 & 3159.20 \\
\hline & EFR5 & 37 & 84.73 & 88.21 & 89.94 & 92.80 & 94.64 & 96.47 & 97.62 & 97.90 & 97.96 \\
\hline & C6 & 37 & 460.50 & 498.80 & 584.40 & 834.80 & 1123.30 & 1451.70 & 2084.60 & 2622.10 & 2692.50 \\
\hline & EFR6 & 37 & 82.24 & 84.97 & 89.17 & 93.13 & 94.45 & 96.31 & 97.67 & 98.54 & 98.59 \\
\hline & $\mathrm{C} 7$ & 37 & 419.10 & 434.40 & 523.90 & 738.30 & 1139.10 & 1728.10 & 2338.20 & 2466.40 & 2544.70 \\
\hline & EFR7 & 37 & 82.40 & 85.19 & 88.59 & 92.44 & 94.97 & 96.53 & 97.46 & 98.10 & 98.29 \\
\hline & SOM & 37 & 95.75 & 97.57 & 98.88 & 99.27 & 99.52 & 99.76 & 99.90 & 100.33 & 100.41 \\
\hline & VIS & 37 & 90.88 & 93.01 & 95.07 & 97.10 & 97.95 & 98.40 & 99.16 & 99.51 & 99.78 \\
\hline & VEST & 37 & 67.39 & 85.16 & 85.77 & 90.25 & 93.08 & 94.74 & 96.72 & 97.29 & 99.25 \\
\hline & DPVD & 37 & 93.78 & 97.86 & 99.41 & 100.80 & 102.28 & 104.98 & 109.08 & 110.79 & 132.21 \\
\hline & DPVE & 37 & 94.04 & 97.17 & 98.89 & 100.91 & 101.81 & 104.54 & 110.11 & 111.21 & 123.26 \\
\hline & DPVT & 37 & 94.26 & 95.23 & 98.05 & 100.19 & 102.48 & 105.33 & 109.29 & 110.58 & 123.49 \\
\hline & IEC & 37 & 84.62 & 88.81 & 91.57 & 93.58 & 95.08 & 96.75 & 97.56 & 97.89 & 98.22 \\
\hline \multirow[t]{22}{*}{$70-89$} & LE & 23 & 7020.60 & 8794.40 & 9681.60 & 11516.00 & 16921.00 & 22094.00 & 25446.00 & 25738.00 & 26310.00 \\
\hline & C1 & 23 & 24.50 & 49.10 & 69.60 & 79.40 & 141.40 & 200.30 & 254.00 & 290.20 & 342.30 \\
\hline & EFR1 & 23 & 98.03 & 98.29 & 98.36 & 98.67 & 99.12 & 99.50 & 99.66 & 99.71 & 99.89 \\
\hline & C2 & 23 & 58.30 & 93.60 & 134.70 & 156.90 & 270.40 & 405.40 & 475.60 & 596.10 & 615.00 \\
\hline & EFR2 & 23 & 95.22 & 96.36 & 97.20 & 97.21 & 98.06 & 99.01 & 99.17 & 99.35 & 99.57 \\
\hline & C3 & 23 & 284.70 & 294.30 & 330.30 & 718.00 & 988.30 & 1205.80 & 1507.90 & 1654.30 & 1940.70 \\
\hline & EFR3 & 23 & 78.52 & 88.53 & 88.62 & 92.09 & 94.13 & 96.37 & 98.04 & 98.09 & 98.47 \\
\hline & C4 & 23 & 755.40 & 1581.50 & 1646.70 & 1846.70 & 2411.10 & 3023.20 & 3298.10 & 3573.60 & 5845.60 \\
\hline & EFR4 & 23 & 65.66 & 67.62 & 74.14 & 77.23 & 85.70 & 90.42 & 92.31 & 92.69 & 94.79 \\
\hline & C5 & 23 & 540.90 & 796.10 & 894.70 & 1173.10 & 1638.60 & 2114.40 & 2617.60 & 2690.90 & 3006.10 \\
\hline & EFR5 & 23 & 77.36 & 78.03 & 81.93 & 86.09 & 90.33 & 93.76 & 94.79 & 96.27 & 96.33 \\
\hline & $\mathrm{C} 6$ & 23 & 730.40 & 787.60 & 881.60 & 1159.30 & 1840.50 & 2120.10 & 2519.30 & 3377.00 & 3953.80 \\
\hline & EFR6 & 23 & 71.35 & 73.78 & 76.69 & 85.39 & 90.92 & 92.48 & 95.22 & 95.87 & 96.36 \\
\hline & C7 & 23 & 524.10 & 614.40 & 795.80 & 1033.30 & 1474.80 & 1950.60 & 2162.90 & 2259.80 & 2433.30 \\
\hline & EFR7 & 23 & 80.08 & 80.13 & 83.16 & 88.37 & 90.49 & 94.67 & 96.00 & 96.33 & 96.39 \\
\hline & SOM & 23 & 97.14 & 97.48 & 97.69 & 98.17 & 99.29 & 99.69 & 99.85 & 99.92 & 99.95 \\
\hline & VIS & 23 & 79.71 & 88.79 & 89.54 & 93.62 & 95.67 & 96.70 & 98.58 & 98.82 & 98.91 \\
\hline & VEST & 23 & 66.65 & 68.31 & 75.43 & 78.32 & 86.97 & 91.45 & 93.02 & 93.14 & 95.31 \\
\hline & DPVD & 23 & 97.49 & 98.59 & 100.14 & 101.11 & 106.43 & 112.63 & 116.12 & 117.82 & 118.07 \\
\hline & DPVE & 23 & 94.80 & 96.25 & 99.12 & 100.15 & 103.96 & 110.73 & 112.79 & 115.51 & 119.92 \\
\hline & DPVT & 23 & 98.28 & 100.01 & 101.68 & 103.25 & 104.95 & 112.58 & 121.94 & 121.97 & 131.32 \\
\hline & IEC & 23 & 77.77 & 82.33 & 83.67 & 88.58 & 90.73 & 93.58 & 95.56 & 96.06 & 96.45 \\
\hline
\end{tabular}

Legenda: $\mathrm{N}$ : voluntários.LE: limite de estabilidade $\left(\mathrm{mm}^{2}\right)$. C1: condição olhos abertos superfície estável(mm²). C2: condição olhos fechados superfície estável(mm²). C3: área elipse de confiança da condição olhos abertos superfície instável( $\left.\mathrm{mm}^{2}\right)$. C4: condição olhos fechados superfície instável(mm²). C5: condição estimulação optocinética para a direita superfície instável( $\left.\mathrm{mm}^{2}\right)$. C6: condição estimulação optocinética para a esquerda superfície instável(mm²). C7: condição estimulação túnel superfície instável((mm²). EFR1: olhos abertos, superfície estável(\%). EFR2: olhos fechados, superfície estável(\%). EFR3: olhos abertos, superfície instável(\%). EFR4: olhos fechados, superfície instável(\%)(\%). EFR5: estimulação optocinética para a direita, superfície instável. EFR6: estimulação optocinética para a esquerda, superfície instável(\%). EFR7: estimulação túnel, superfície instável(\%). SOM: somatossensorial(\%). VIS: visual(\%). VEST: vestibular(\%). DPVD: dependência visual optocinético para direita(\%). DPVE: dependência visual optocinético para esquerda(\%). DPVT: dependência visual túnel(\%). IEC: índice de equilíbrio composto(\%).

\section{DISCUSSÃO}

O posturógrafo brasileiro Horus é um equipamento inovador e de baixo custo frente aos existentes no mercado internacional e foi criado de acordo com a demanda de profissionais da área de diagnóstico e tratamento do equilíbrio, pois era necessário um protocolo de avaliação com valores de referência da população brasileira.

$\mathrm{Na}$ posturografia estática, registram-se as oscilações corporais quando o indivíduo permanece em postura ereta, 
sem movimento. Já na posturografia dinâmica, o registro é realizado após uma perturbação aplicada ao sujeito ${ }^{(7)}$. Em nosso estudo, foram incluídos procedimentos que envolvem o controle postural estático e dinâmico, por meio do teste de limite de estabilidade e também por meio de superfície instável criada pelo uso de uma almofada, permitindo avaliar a contribuição do sistema proprioceptivo no equilíbrio. Portanto, adotamos a nomenclatura "Posturografia estática com provas dinâmicas". Pesquisa realizada com 89 sujeitos $^{(15)}$, sendo 59 sadios e $30 \mathrm{com}$ alterações vestibulares periféricas, usou a posturografia estática com provas dinâmicas. Os autores observaram que os sujeitos sadios apresentaram menores oscilações e, por consequência, valores de limite de estabilidade maiores e resultados nos testes de controle rítmicos e direcional melhores do que os indivíduos enfermos. Concluiu-se que a posturografia estática com provas dinâmicas é um método viável de avaliação, capaz de auxiliar o diagnóstico de alterações vestibulares.

De acordo com os testes estatísticos aplicados, este estudo mostrou que diferentes faixas etárias e gêneros apresentaram diferenças estatisticamente significantes. Comparando cada grupo de faixa etária, notou-se que os dados quantitativos mudaram, sugerindo mudanças no controle postural com o avanço da idade mesmo em indivíduos hígidos. As mesmas faixas etárias foram adotadas para definir dados normativos para a posturografia computadorizada dinâmica Equitest da Neurocom e Bertec Dynamic CPD plataform ${ }^{(11)}$. Também outros autores ${ }^{(1,4,7)}$ perceberam que a faixa etária influenciou diretamente os resultados da estabilometria. Ao estudar idosos, confirmaram que a idade deteriora o equilíbrio, aumentando o risco de quedas. Observaram alterações significativas em idosos enquanto realizavam testes que suprimiam a visão e propriocepção, justificando o alto índice de quedas e reforçando um distúrbio vestibular como alteração de base.

Em relação ao teste de limite de estabilidade, com o aumento da idade, verificou-se uma tendência a se reduzir sua área, o que poderia explicar, em parte, o aumento da incidência de quedas na terceira idade em indivíduos sintomáticos. Além disso, comparando a área do limite de estabilidade entre os gêneros, constataramse valores maiores para indivíduos do gênero masculino. Essa diferença pode ser explicada pela estatura dos pacientes do gênero masculino que, em geral, foi superior à do grupo feminino, já que, quanto mais alto o indivíduo, maior sua base de sustentação. Pesquisas anteriores ${ }^{(1,4,7)}$ apresentaram conclusões análogas.

Os valores percentis foram estudados considerando as velocidades anteroposterior e médio-lateral, além da banda de frequências que abrange $80 \%$ da potência do sinal em cada eixo. Autores ${ }^{(14)}$ revelaram a importância de estudar o controle postural relacionado à velocidade de oscilação corporal e à área de elipse de confiança pela posturografia estática com o equipamento Balance Rehabilitation Unit (BRUTM), tendo analisado 30 pacientes com doença de Ménière definida e 40 indivíduos hígidos. Foram encontradas anormalidades na área da elipse de confiança e na velocidade de oscilação corporal nos pacientes com doença de Ménière. Quanto menor a frequência de oscilação do corpo, menores são as características dinâmicas na manutenção do equilíbrio. Para frequências de até $0,2 \mathrm{~Hz}$, cerca de $10 \%$ da oscilação do $\mathrm{CP}$ não representam a oscilação do CG, mas sim acelerações de segmentos corporais. A banda de frequências que abrange $80 \%$ da potência espectral é a que melhor caracteriza as alterações do sistema de controle postural ${ }^{(10)}$.

Analisando os dados do teste de integração sensorial, independentemente da faixa etária, notou-se o aumento da área de elipse de confiança, da velocidade média e dos valores da banda de frequência FB1, bem como a redução do EFR, à medida que as entradas sensoriais visuais e somatossensoriais foram retiradas/distorcidas. Isso pode fornecer informações sobre o modo como os indivíduos com distúrbios do equilíbrio corporal realizam o processo de integração sensorial, permitindo um diagnóstico mais preciso das alterações do controle postural. Autores de outros estudos ${ }^{(3-5,12-14,16-18)}$ observaram o mesmo efeito.

$\mathrm{O}$ estudo $^{(19)}$ com tecnologia brasileira e também de baixo custo chamada "posturografia foam-laser", para a realização de testes de organização sensorial com o uso de uma almofada de média densidade, concluiu ser um método simples, que produziu análises de organização sensorial que são comparáveis àquelas obtidas com o equipamento para posturografia dinâmica. A técnica utilizada em nosso trabalho teve o mesmo objetivo, porém com uma plataforma de força e um sistema computadorizado que facilitou a obtenção das respostas, a mensuração de grandezas que não se poderia obter com a simples observação, a repetibilidade das medidas e a organização das informações coletadas num sistema previamente calibrado.

A posturografia Horus tem semelhanças com a maioria dos posturógrafos $^{(1,3-5,9-16-18)}$. O software registra os sinais do CP durante cada teste, extrai parâmetros numéricos nos domínios do tempo e da frequência, além de incorporar estrutura para viabilizar a comparação desses parâmetros contra valores de referência, apresentando gráficos que facilitam a compreensão das respostas da análise sensorial. A partir do presente estudo, os valores obtidos podem ser aproveitados pelos usuários do sistema Horus para auxiliar a identificação de pacientes que apresentem comprometimento de um ou mais dos sistemas que compõem o equilíbrio, desde que se execute o protocolo proposto aqui.

A posturografia é um instrumento importante para complementar a avaliação do equilíbrio, mas os equipamentos disponíveis até aqui eram de difícil acesso, pelas razões já expostas e de acordo com vários outros trabalhos ${ }^{(3,4,6,6,8,11,14-18)}$, fato que impossibilitava sua disseminação na prática clínica. A partir do protocolo proposto neste trabalho e dos valores de referência estabelecidos, novas pesquisas poderão ser realizadas abordando diferentes tipos de doença e alterações, tanto vestibulares quanto visuais e somatossensoriais, concorrendo para a melhor avaliação e compreensão da evolução das doenças do equilíbrio.

\section{CONCLUSÃO}

Foi possível estabelecer um protocolo para investigar o equilíbrio corporal com valores de referência em função de diversas faixas etárias e gêneros.

\section{REFERÊNCIAS}

1. Patti A, Bianco A, Şahin N, Sekulic D, Paoli A, Iovane A, et al. Postural control and balance in a cohort of healthy people living in Europe: an 
observational study. Medicine (Baltimore). 2018;97(52):e13835. http:// dx.doi.org/10.1097/MD.0000000000013835. PMid:30593180.

2. Reynard F, Christe D, Terrier P. Postural control in healthy adults: determinants of trunk sway assessed with a chest-worn accelerometer in 12 quiet standing tasks. PLoS One. 2019;14(1):e0211051. http://dx.doi. org/10.1371/journal.pone.0211051. PMid:30673753.

3. Quitschal RM, Fukunaga JY, Ganança MM, Caovilla HH. Evaluation of postural control in unilateral vestibular hypofunction. Braz J Otorhinolaryngol. 2014;80(4):339-45. http://dx.doi.org/10.1016/j.bjorl.2014.05.015. PMid:25183185.

4. Mecenas TS. Aspectos normativos da posturografia dinâmica computadorizada no adulto e no idoso [dissertação]. Ribeirão Preto: Faculdade de Medicina, Universidade de São Paulo; 2014 [citado em 2020 Abr 23]. Disponível em: https://teses.usp.br/teses/disponiveis/17/17150/tde-14032014-112108/ publico/Dissertacao.pdf

5. Pimentel BN, Santos VAV Fa. Ocorrência de condições psiquiátricas, uso de psicotrópicos e sua relação com o equilíbrio postural em sujeitos com tontura. CoDAS. 2019;31(3):e20180111. http://dx.doi.org/10.1590/23171782/20182018111. PMid:31271579.

6. Fukunaga JY, Quitschal R, Doná F, Ferraz HB, Ganança MM, Caovilla HH. Postural control in Parkinson's disease. Braz J Otorhinolaryngol. 2014;80(6):508-14. http://dx.doi.org/10.1016/j.bjorl.2014.05.032. PMid:25457071.

7. Duarte M, Freitas SM. Revisão sobre posturografia baseada em plataforma de força para avaliação do equilíbrio. Braz J Phys Ther. 2010;14(3):183-92. http://dx.doi.org/10.1590/S1413-35552010000300003.

8. Oda DTM, Ganança CF. Posturografia dinâmica computadorizada na avaliação do equilíbrio corporal de indivíduos com disfunção vestibular. Audiol Commun Res. 2015;20(2):89-95. http://dx.doi.org/10.1590/S231764312015000200001469 .

9. Bruniera JRZ, Camiloti JF, Penha OM, Franco PPR, Silva RA Jr, Marchiori LLM. Análise comparativa do equilíbrio postural pela posturografia em pacientes com vertigem isolada ou associada com perda auditiva. Audiol Commun Res. 2015;20(4):321-6. http://dx.doi.org/10.1590/2317-64312014-1534.

10. Baratto L, Morasso PG, Re C, Spada G. A new look at posturographic analysis in the clinical context: sway-density versus other parameterization techniques. Motor Control. 2002;6(3):246-70. http://dx.doi.org/10.1123/ mcj.6.3.246. PMid:12122219.

11. Trueblood PR, Rivera M, Lopez C, Bentley C, Wubenhorst N. Age-based normative data for a computerized dynamic posturography system that uses a virtual visual surround environment. Acta Otolaryngol. 2018;138(7):597602. http://dx.doi.org/10.1080/00016489.2018.1429653. PMid:29390922.

12. Kalron A, Nitzani D, Achiron A. Static posturography across the EDSS scale in people with multiple sclerosis: a cross sectional study. BMC
Neurol. 2016;16(1):70. http://dx.doi.org/10.1186/s12883-016-0603-6. PMid:27206921.

13. Van Ombergen A, Lubeck AJ, Van Rompaey V, Maes LK, Stins JF, Van de Heyning $\mathrm{PH}$, et al. The effect of optokinetic stimulation on perceptual and postural symptoms in visual vestibular mismatch patients. PLoS One. 2016;11(4):e0154528. http://dx.doi.org/10.1371/journal.pone.0154528. PMid:27128970.

14. Cusin FS, Ganança MM, Ganança FF, Ganança CF, Caovilla HH. Balance Rehabilitation Unit (BRU) posturography in Menière's disease. Braz J Otorhinolaryngol. 2010;76(5):611-7. http://dx.doi.org/10.1590/S180886942010000500013. PMid:20963345.

15. Balaguer García R, Pitarch Corresa S, Baydal Bertomeu JM, Morales Suárez-Varela MM. Static posturography with dynamic tests. Usefulness of biomechanical parameters in assessing vestibular patients. Acta Otorrinolaringol Esp. 2012;63(5):332-8. http://dx.doi.org/10.1016/j. otorri.2012.03.006. PMid:22633316.

16. Micarelli A, Liguori C, Viziano A, Izzi F, Placidi F, Alessandrini M. Integrating postural and vestibular dimensions to depict impairment in moderate-to-severe obstructive sleep apnea syndrome patients. J Sleep Res. 2017;26(4):487-94. http://dx.doi.org/10.1111/jsr. 12516 PMid:28318060.

17. Söhsten E, Bittar RS, Staab JP. Posturographic profile of patients with persistent postural-perceptual dizziness on the sensory organization test. J Vestib Res. 2016;26(3):319-26. http://dx.doi.org/10.3233/VES-160583. PMid:27392836.

18. Ongun N, Atalay NS, Degirmenci E, Sahin F, Bir LS. Tetra-ataxiometric posturography in patients with migrainous vertigo. Pain Physician. 2016;19(1):E87-95. PMID: 26752496.

19. Castagno LA. A new method for sensory organization tests: the foamlaser dynamic posturography. Rev Bras Otorrinolaringol (Engl Ed). 1994;60(4):287-96.

20. Lim YH, Kim JS, Lee HW, Kim SH. Postural instability induced by visual motion stimuli in patients with vestibular migraine. Fron Neurol. 2018;9:433. http://dx.doi.org/10.3389/fneur.2018.00433. PMid:29930534.

\section{Contribuição dos autores}

LKN participou da idealização do estudo, coleta, análise e interpretação dos dados e redação do artigo; GDR participou da coleta, análise e interpretação dos dados e redação do artigo; TSAS participou da coleta de dados e correção do artigo; FAQR participou na condição de orientação do artigo, análise e interpretação dos dados e redação do artigo; PLS participou na condição de orientação do artigo, coleta, análise e interpretação dos dados e redação do artigo 